\title{
Carter Constant and Angular Momentum
}

\author{
Sajal Mukherjee and K. Rajesh Nayak, \\ IISER Kolkata, Mohanpur, India..
}

July 8, 2015

\begin{abstract}
We investigate the Carter-like constant in the case of a particle moving in a nonrelativistic dipolar potential. This special case is a missing link between Carter constant in stationary axially symmetric spacetimes such as Kerr solution and its possible Newtonian counterpart. We use this system to carry over the definition of angular momentum from the Newtonian mechanics to the relativistic stationary axially symmetric spacetimes.
\end{abstract}

\section{Introduction}

The Carter constant is one of the non-trivial integrals of motion for a particle moving in a stationary axially symmetric spacetimes (SASS) such as Kerr solution [1]. Though there have been several studies on the physical interpretation, a self-consistence understanding of this integral of motion has been a problem. In the case of simpler spherically symmetric solutions, the Carter constant reduces to the square of the angular momentum $\left(L^{2}=\vec{L} \cdot \vec{L}\right)[2$. One of the obstacles in understanding the Carter constant is the complexity of the SASS, because of the physical effects such as frame-dragging [3]. The phenomenon frame-dragging is essentially a pure general relativistic effects that do not have any Newtonian analogy. For a simpler understanding and to investigate non-trivial properties of the Carter constant, it would be good to have a solution to Einstein equations which is axially symmetric and static. However, attemptst to find such solutions, allowing Carter constant are not very successful [4]. Another approach is to look for a Newtonian system that would give rise to a non-trivial Carter-like constant. Recently, Will [5] has given Carter-like constant in the Newtonian dynamics. Here, we take a similar approach and show that Carter-like constant exists for a charged particle moving in a field of an electric dipole. Perhaps, this case can be used for better understanding the physics of Carter constant in general. We also look at the Carter constant in the case of relativistic rotating frame. In this standard example, the Carter constant reduces to $L^{2}$.

The angular momentum vector is a purely Newtonian concept, and it plays an important roll in understanding physics of a rotating system. However, it does not have any covariant 
definition, and this has been one of the problems in the general theory of relativity. The angular momentum vector, because of the interrelation between it's components, only two independent scalar quantities can be constructed. Conventionally they are the azimuthal component of the angular momentum, $L_{z}$ and square of the angular momentum, $L^{2}$. Until a valid covariant definition of angular momentum is established, one may use $L_{z}$ and $L^{2}$ for understanding the role of angular momentum in the relativistic rotating systems. In the case spherical symmetry, both $L_{z}$ and $L^{2}$ are conserved, and it is very easy to identify them. However, in the case SASS, $L_{z}$ is conserved and in general $L^{2}$ need not be conserved. In such case, there is no straightforward way to identify $L^{2}$. It is here we use the Carter constant with its Newtonian counterpart to define $L^{2}$ in SASS. One of the interesting special case would be, a SASS that allows $L^{2}$ as a conserved quantity. We show that only spherically symmetric and flat spacetime allow such possibility.

The central point of this article is around a theorem given by Carter [6], it states, for a Hamiltonian the form:

$$
\mathcal{H}=\frac{1}{2} \frac{H_{r}+H_{\theta}}{U_{r}+U_{\theta}}
$$

where, $U_{r}, U_{\theta}$ are functions of single variable $r, \theta$ respectively. The function $H_{r}$ is function of $r$ and canonical momenta other than $p_{\theta}$, while $H_{\theta}$ is function of $\theta$ and canonical momenta other than $p_{r}$, then

$$
\mathcal{K}=\frac{U_{r} H_{\theta}-U_{\theta} H_{r}}{U_{r}+U_{\theta}}
$$

is a constant of motion. This integral of motion $\mathcal{K}$ was named after Carter. This theorem is a generalised version of Stäckel theorem, which put the constraint on the form of potentials giving rise to conserved quantity through the Hamilton-Jacobi theory [7].

In this article, we apply the Carter's theorem to three examples. In the first case, for a charged particle moving in the field of an electric dipole. This system is a well understood Newtonian axially symmetric system. In section-3, we apply it to a particle moving in a rotating frame. Finally in the section-4, we apply it to the case of SASS and Kerr spacetime as a special case. We show that the Newtonian definition of $L^{2}$ can be carried over to SASS. Finally, we close the article with a brief concluding remark.

\section{Carter-Stäckel constant for a dipolar field}

We apply the Carter theorem for the motion of a charged particle in an axially symmetric electrostatic field. The Most suitable coordinates to study axially symmetric system is the spherical polar coordinates $\{r, \theta, \phi\}$. The non-relativistic Hamiltonian in the spherical polar coordinate takes the form:

$$
\mathcal{H}=\frac{1}{2 m r^{2}}\left\{\left(r p_{r}\right)^{2}+\left(p_{\theta}\right)^{2}+\frac{1}{\sin ^{2} \theta}\left(p_{\phi}\right)^{2}+2 m r^{2} \Phi(r, \theta)\right\} .
$$


We use the following choice of decomposition for $\mathcal{H}$, in terms of $\left\{H_{r}, H_{\theta}, U_{r}, U_{\theta}\right\}$ :

$$
\begin{aligned}
U_{r}=r^{2}, & U_{\theta}=0, \\
H_{r}=r^{2} p_{r}^{2} & H_{\theta}=\left(p_{\theta}\right)^{2}+\frac{1}{\sin ^{2} \theta}\left(p_{\phi}\right)^{2}+2 r^{2} \Phi(r, \theta) .
\end{aligned}
$$

Form separability point of view, we required that $\Phi(r, \theta)=\frac{1}{r^{2}} g(\theta)$. Where $g(\theta)$ can be any arbitrary function of $\theta$ [8]. This form of potential is known as Stäckel potential [8]. From the physics point of view, the choice of potential is:

$$
\Phi(r, \theta)=\frac{a}{r^{2}} \cos \theta .
$$

In the above, $a$ is a constant, and the potential is due to a point dipole. The Newtonian gravity itself does not allow dipolar field; we can consider an example from classical electrodynamics, a point charge particle in the field of point electric dipole. The corresponding conserved quantity is:

$$
\mathcal{K}_{\text {dipole }}=H_{\theta}=\left[p_{\theta}^{2}+\frac{1}{\sin ^{2} \theta} p_{\phi}^{2}\right]+2 a \cos \theta=L^{2}+2 a \cos \theta .
$$

We may note that $p_{\phi}=L_{z}$, the azimuthal component of the angular momentum. When the field is spherically symmetric, i.e. if $a=0$, the Carter constant reduces to $L^{2}$. The dipolar field gives the lowest non-trivial contribution to the Carter-Stäckel constant. It is the case where conservation of $L^{2}$ is not valid. Since, in the Newtonian mechanics, angular momentum is defined unambiguously, we can identify the two terms, the first one as $L^{2}$ and the second one as effective potential for motion along $\theta$.

The motion of a charged particle in electric dipole field has been well studies starting from Turner and Fox [9]. However, the existence of an additional integral of motion was first explicitly emphasised by Gutierres-Lopez, Castellanos-Moreno and Rosas-Burgos [10], without dwelling on its close association with the Carter constant. They have given an indepth analysis of the effect of this constant on the motion of charge particle. In particular, we would like to draw attention to the maximum angle subtended by the charged particle while moving on a sphere $(r=$ constant $)$ for a given value of Carter constant. Similar behaviour is also exhibited by a particle moving in the Kerr spacetime [11.

The precession is one of the consequences of non-conservation of angular momentum. In our case, the precession equation can be obtained by taking the derivative of Eq (6), resulting in:

$$
\vec{L} \cdot \dot{\vec{L}}=-\beta q \sin \theta \dot{\theta}
$$

This relation gives the precession of angular momentum for a particle orbiting around a dipole, implying that the motion of the particle is not confined to a single plane. 


\section{Rotating Frame}

In this section, we apply the Carter's theorem to a particle moving in a relativistic rotating frame. The spacetime metric with a rotation along the $z$-axis with constant angular velocity $\omega$ can be written as:

$$
d s^{2}=-\left(1-\omega^{2} r^{2} \sin ^{2} \theta\right) d t^{2}-2 \omega r^{2} \sin ^{2} \theta d t d \phi+r^{2} \sin ^{2} \theta d \phi^{2}+d r^{2}+r^{2} d \theta^{2} .
$$

The spacetime metric components are functions of the coordinates and will have at least one off-diagonal component. However, the absence of gravity is reflected as vanishing Riemann curvature, i.e., $R_{a b c d} \equiv 0$. In this case, the Hamiltonian for a particle can be written as:

$$
\mathcal{H}=\frac{1}{r^{2}}\left[-r^{2} p_{t}^{2}-2 \omega r^{2} p_{t} p_{\phi}+\left(\frac{1}{\sin ^{2} \theta}-\omega^{2} r^{2}\right) p_{\phi}^{2}+r^{2} p_{r}^{2}+p_{\theta}^{2}\right] .
$$

It is straight forward to apply the Carters theorem, resulting in:

$$
\mathcal{K}_{\text {rotate }}=\left[p_{\theta}^{2}+\frac{1}{\sin ^{2} \theta} p_{\phi}^{2}\right]=L^{2} .
$$

In this case, both $L_{z}$ and $L^{2}$ are conserved. Irrespective of the rotation of the frame itself here the Carter constant reduces to $L^{2}$. We can easily extend the dipole potential given in the section-2 to a rotating frame. This case will not change the overall scenario, as the rotation does not affect potential. However, the rotating systems in the general relativity introduce more complexity that we discuss in the next section.

\section{Stationary Axially Symmetric Spacetimes}

In this section, we apply the Carter's theorem to the case of a particle moving in SASS. Here, we closely follow the Carter's work [6]. It has been shown that, for a spacetime to allow Carter constant, it is sufficient that the spacetime metric takes the following form[6]:

$$
d s^{2}=\Sigma\left\{\frac{d r^{2}}{\Delta_{r}}+\frac{d \mu^{2}}{\Delta_{\mu}}\right\}+\frac{1}{\Sigma}\left\{\Delta_{\mu}\left[C_{r} d t-Z_{r} d \phi\right]^{2}-\Delta_{r}\left[C_{\mu} d t-Z_{\mu} d \phi\right]^{2}\right\} .
$$

In the above, $\Sigma=\left(C_{\mu} Z_{r}-C_{r} Z_{\mu}\right), C_{r}$ and $C_{\mu}$ are constants. The parameters $Z_{\mu}$ and $\Delta_{\mu}$ are functions of single variable $\mu=\cos \theta$. While, the parameters $Z_{r}$ and $\Delta_{r}$ are functions of $r$ only [6]. These functions can be obtained by solving the Einstein equations. For example, in the case of the Kerr solution [12, these parameters are given by:

$$
\begin{aligned}
C_{\mu}=1, & C_{r}=a, \\
Z_{r}=r^{2}+a^{2}, & Z_{\mu}=a\left(1-\mu^{2}\right), \\
\Delta_{r}=r^{2}-2 M r+a^{2}, & \Delta_{\mu}=1-\mu^{2} .
\end{aligned}
$$


The metric given in Eq. (11), allows a time Killing vector field $\xi^{a}$ and a rotational Killing vector $\eta^{a}$. The corresponding conserved quantities are, energy $E=p_{a} \xi^{a}$ and the azimuthal component of angular momentum $L_{z}=p_{a} \eta^{a}$.

The Newman-Penrose formalism is very useful in studying the Carter constant. For the metric given in Eq. (11), components of the complex null tetrad are given by [13]:

$$
\begin{aligned}
l^{a} & =\frac{1}{\Delta_{r}}\left(Z_{r}, \Delta_{r}, 0, C_{r}\right), \\
n^{a} & =\frac{1}{2 \Sigma}\left(Z_{r},-\Delta_{r}, 0, C_{r}\right), \\
m^{a} & =\frac{1}{\sqrt{2 \Sigma}}\left(\frac{i Z_{\mu}}{\sqrt{\Delta_{\mu}}}, 0, \sqrt{\Delta_{\mu}}, \frac{i C_{\mu}}{\sqrt{\Delta_{\mu}}}\right) .
\end{aligned}
$$

The null vectors $l^{a}$ and $n^{a}$ are principal null directions [14, 15]. The spacetime metric in terms of the null tetrad is given by:

$$
g^{a b}=-\left(l^{a} n^{b}+l^{b} n^{a}-m^{b} \bar{m}^{a}-m^{a} \bar{m}^{b}\right) .
$$

In the above, $\bar{m}^{a}$ is the complex conjugate of $m^{a}$. In this case, the Hamiltonian $\mathcal{H}$, takes the form:

$$
\mathcal{H}=\frac{1}{\Sigma}\left\{\Delta_{\mu} p_{\mu}^{2}+\Delta_{r} p_{r}^{2}+\Delta_{\mu}^{-1}\left[C_{\mu} p_{\phi}+Z_{\mu} p_{t}\right]^{2}-\Delta_{r}^{-1}\left[C_{r} p_{\phi}+Z_{r} p_{t}\right]^{2}\right\}
$$

It is straightforward to compute the Carter constant using Eq. (11) and Eq. (2) 6]. After some simplification, we get:

$$
\mathcal{K}_{\text {sass }}=\left[\Delta_{\mu} p_{\mu}^{2}+\frac{C_{\mu}^{2}}{\Delta_{\mu}} p_{\phi}^{2}\right]+\left[\frac{Z_{\mu}^{2}}{\Delta_{\mu}} E^{2}-2 \frac{C_{\mu} Z_{\mu}}{\Delta_{\mu}} L_{z} E-\frac{1}{2} C_{r} Z_{\mu}\right]
$$

Here, we use normalising condition $\mathcal{H}=g^{a b} p_{a} p_{b}=-\frac{1}{2}$. Interestingly, this equation looks very similar to the dipole case as given in Eq.(6). In the above equation we have, first part which is quadratic in momenta of angular motion, i.e. along $\mu$ and $\phi$. In analogy with Eq. (6), we may recognise it as $L^{2}$. The second part, depend only on $\mu$ and constants of motion such as $E$ and $L_{z}$. It is associated with the effective potential for motion along $\mu$. From this close similarity, we define $L^{2}$ in the case of axially symmetric stationary spacetimes as,

$$
L^{2}=\left[\Delta_{\mu} p_{\mu}^{2}+\frac{C_{\mu}^{2}}{\Delta_{\mu}} p_{\phi}^{2}\right]
$$

It is just the Newtonian definition carried over to the relativistic case, which is valid for SASS with the metric given by the Eq.(15). The above equation gives the definition of $L^{2}$ in terms of generalised momenta, $p_{\theta}$ and $p_{\phi}$. It should be noted that form of $p_{\theta}$ and $p_{\phi}$ 
might be different in each case. Here, we would like to remind that, in the case of SASS, the $p_{a}$ in terms of $\dot{x}^{a}$ are given by:

$$
\begin{array}{ll}
p_{t}=g_{t t} \dot{t}+g_{t \phi} \dot{\phi} & =\frac{\Delta_{\mu} C_{r}^{2}-\Delta_{r} C_{\mu}^{2}}{\Sigma} \dot{t}+\frac{\Delta_{r} C_{\mu} Z_{\mu}-\Delta_{\mu} C_{r} Z_{r}}{\Sigma} \dot{\phi} \\
p_{r}=g_{r r} \dot{r} & =\frac{\Sigma}{\Delta_{r}} \dot{r} \\
p_{\mu}=g_{\mu \mu} \dot{\mu} & =\frac{\Sigma}{\Delta_{\mu}} \dot{\mu}, \\
p_{\phi}=g_{t \phi} \dot{t}+g_{\phi \phi} \dot{\phi} & =\frac{\Delta_{r} C_{\mu} Z_{\mu}-\Delta_{\mu} C_{r} Z_{r}}{\Sigma} \dot{t}+\frac{\Delta_{\mu} Z_{r}^{2}-\Delta_{r} Z_{\mu}^{2}}{\Sigma} \dot{\phi} .
\end{array}
$$

Though $L^{2}$ takes the simpler form similar to Newtonian case, the complexity of relativistic rotation is still maintained. In the case of Kerr spacetime, from Eq. (12) and Eq. (16), we get this well known expression for Carter constant:

$$
\mathcal{K}_{\text {kerr }}=\left[p_{\theta}^{2}+\frac{1}{\sin ^{2} \theta} p_{\phi}^{2}\right]+a^{2} \sin ^{2} \theta E^{2}-2 a E L z-\frac{a^{2} \sin ^{2} \theta}{2} .
$$

It should be noted that, $L^{2}=\left[p_{\theta}^{2}+\frac{1}{\sin ^{2} \theta} p_{\phi}^{2}\right]$, comes from Eq. (17) and is not as a result of setting parameter $a \rightarrow 0$.

In the past, there have been attempts to define angular momentum through the Floyd tensor [16, 17]. However, we take a simpler approach, by defining the quantities $L_{z}$ and $L^{2}$. As has been mentioned earlier, these are the two physically important scalar quantities connected with angular momentum vector. In general, $L^{2}$ is not conserved, if the separability conditions are satisfied as per Carter's theorem, we have additional conserved quantity $\mathcal{K}_{\text {sass. }}$. Physically it indicates that, in general, the motion of a free particle in SASS is not confined to a plane. Here, we have obtained this definition of $L^{2}$ from analogy and intuition. In the next section, we arrive at the same result with a more formal approach.

\subsection{Relation with Killing Tensor}

In this section, we approach the definition of $L^{2}$ in SASS using the Killing tensor. The Carter constant can also be obtained from the Killing tensor [18. The Killing tensor in terms of the principal null vectors for SASS is given by,

$$
K^{a b}=\Sigma\left(l^{a} n^{b}+l^{b} n^{a}\right)+C_{\mu} Z_{r} g^{a b}
$$

For a particle moving along geodesic with four-momentum $p^{a}, \mathcal{K}=K^{a b} p_{a} p_{b}$ is conserved and can be shown to be Carter constant given by Eq. (16). We show that the Killing tensor given in Eq. (21), can be casted in a following alternative form:

$$
K^{a b}=\Sigma\left(m^{a} \bar{m}^{b}+m^{b} \bar{m}^{a}\right)+C_{r} Z_{\mu} g^{a b} .
$$


It is straightforward to show that the $K^{a b}$ indeed a Killing tensor. After some simplification it can be shown that the conserved quantity $\mathcal{K}=K^{a b} p_{a} p_{b}$ is same as the one given in Eq. (16). Because of the normalisation condition $g^{a b} p_{a} p_{b}=-\frac{1}{2}$, the last term is Eq. (21) independent of $p_{a}$ and can not contribute to $L^{2}$. In the definition of $L^{2}$, we consider only the first term, which is:

$$
L^{a b}=\Sigma\left(m^{a} \bar{m}^{b}+m^{b} \bar{m}^{a}\right)
$$

We project this onto two-surface orthogonal to the surface formed by $\chi^{a}$ and $\gamma^{a}$. Where,

$$
\chi^{a}=\xi^{a}-\frac{\xi^{p} \eta_{p}}{\eta^{q} \eta_{q}} \eta^{a} \quad \text { and } \quad \gamma^{a}=(0,1,0,0) .
$$

The vector field $\gamma^{a}$ is along the radial direction. In SASS, the time like-Killing vector $\xi^{a}$ and rotational Killing vector $\eta^{a}$ are surface forming [20. However, they are not orthogonal, i.e., $\xi^{a} \eta_{a} \neq 0$. Besides, if spacetime satisfy the condition of orthogonal transitivity [19], $r-\theta$ surfaces at every point in the spacetime are orthogonal to $\xi-\eta$ surfaces. As $\xi^{a}$ and $\eta^{a}$ are not orthogonal, we span the same surface with the vector field $\chi$ and $\eta$. The vector field $\chi^{a}$ is not a Killing vector field and referred as quasi-Killing vector field [20]. It has several interesting properties, it is hypersurface orthogonal and can be taken as frame closest to the Newtonian global rest frame in SASS [21]. Furthermore, they are also referred as zero angular momentum observers ( ZAMO ) because observers following along $\chi$ experiences no precession or has zero angular momentum [22]. By using this projection, we ensure that no total angular momentum is lost during the projection operation and remain close to a possible Newtonian description. The projection operator onto a $\theta-\phi$ surface is given by,

$$
h^{a b}=g^{a b}-\frac{1}{\chi^{p} \chi_{p}} \chi^{a} \chi^{b}-\frac{1}{\gamma^{q} \gamma_{q}} \gamma^{a} \gamma^{b}
$$

With these, the $L^{2}$ can be defined as:

$$
L^{2}=L^{a b} h_{a}^{k} h_{b}^{l} p_{k} p_{l}
$$

where $L^{a b}$ is given by Eq. (22). It is easy to show this takes the same form given in the Eq. (17).

\subsection{Special cases}

Here, we look for a special case of SASS in which $L^{2}$ is conversed. We start with the case $Z_{\mu}=0$, for which $\mathcal{K}_{\text {sass }}$ reduces to just $L^{2}$. These are a possible set of axially symmetric system that conserves $L^{2}$. For this case, the spacetime metric takes the following form:

$$
d s^{2}=\frac{-1}{C_{\mu} Z_{r}}\left(\Delta_{r} C_{\mu}^{2}-\Delta_{\mu} C_{r}^{2}\right) d t^{2}-2 \frac{\Delta_{\mu} C_{r}}{C_{\mu}} d t d \phi+\frac{\Delta_{\mu} Z_{r}}{C_{\mu}} d \phi^{2}+\frac{C_{\mu} Z_{r}}{\Delta_{r}} d r^{2}+\frac{C_{\mu} Z_{r}}{\Delta_{\mu}} d \mu^{2}
$$


We look for source free solutions to the Einstein's equation satisfied by the above metric. The significant constraint comes from $G_{(\mu)}^{(\mu)}$ component of the Einstein tensor. The equation $G_{(\mu)}^{(\mu)}=0$ implies:

$$
\left(\frac{d Z_{r}}{d r}\right)^{2}\left(C_{\mu}^{2} \Delta_{r}-C_{r}^{2} \Delta_{\mu}\right)+2 C_{\mu}^{2} Z_{r}\left(Z_{r} \frac{d^{2} \Delta_{r}}{d r^{2}}-\frac{d Z_{r}}{d r} \frac{d \Delta_{r}}{d r}\right)=0
$$

$\Delta_{\mu}$ is the only parameter depending on the variable $\mu$. There are two ways obtaining a self-consistent solution:

1. $C_{r}=0$ : In this case, it is straightforward to show that spacetime is spherically symmetric, resulting in the known result $\mathcal{K}=L^{2}$.

2. $C_{r} \neq 0$ : This can be shown to result in the condition that the Riemann tensor $R_{a b c d}=0$, i.e., the space-time is flat. This case may include cases such as rotating frame has given earlier or any other transformation resulting in the metric of the form given in Eq.(26).

Leading to a completely consistent cases of $L^{2}$ conservation for the source free solutions 4 , 23 .

\section{Discussions}

A particle moving in a dipolar field serves as a good Newtonian system for understanding the Carter constant. We have shown that the form of the Newtonian Carter-Stäckel constant has interestingly close similarity with its counterpart in fully relativistic SASS such as Kerr solution. This Analogy allows us carry over the definition of $L^{2}$ from Newtonian mechanics to SASS. We would like to emphasise that only form of the metric is important for this formalism, it does not depend on the nature of the source or asymptotic conditions. Because it is possible to have Carter-like constant for SASS which are not asymptotical flat [17]. More formally, the $L^{2}$ can be defined using Killing tensor and its projection onto a space orthogonal to the space formed by the ZAMO and radial vector. In the case of source free solutions, we also have shown that only spacetimes admitting conservation of $L^{2}$ are: spherically symmetric system and the spacetime with zero Riemann curvature.

\section{Acknowledgments}

We wish to thank the Visiting Associateship programme of Inter-University Centre for Astronomy and Astrophysics (IUCAA), Pune. A part of this work was carried out during the visit to IUCAA under this programme. 


\section{References}

[1] Carter B, Phys. Rev. 174, 1559(1968).

[2] De Felice F. and Preti G,Class. Quantum Grav. 16,2929 (1999).

K. Rosquist, T. Bylund and L. Samuelsson, Int.Journal of Mod.Phys.D 18,429(2009).

[3] Lense J. and Thirring H, Phys. Z. 19, 156(1918).

Lense J. and Thirring H, Phys. Z. 19, 33(1918).

Lense J. and Thirring H, Phys. Z. 22, 29(1921).

[4] Mirshekari S. and Will C.W, Class.Quant.Grav. 27, 235021(2010).

[5] Will C.M, Phys. Rev. Lett. 102 :061101, (2009).

[6] Cater B, "Black Holes", ed. De Witt C and De Witt B S, Gordon and Breach NewYork(1973).

[7] Stäckel, "Compt. Rendus Acad. Sci" 116, 1284(1893).

Pars L.A., The American Mathematical Monthly, 56 , 394(1949).

[8] N.C. Rana and P. C. Joag., "Classical Mechanics", Tata McGraw-Hill Publishing Company Limited(1991).

[9] Turner J. E., Anderson V. E. and Fox K, Phys. Rev. 174, 81(1968).

Turner J.E. and Fox K, J. Phys. A: Gen. Phys. 1,118(1968).

[10] Gutierres-Lopez S, Castellanos-Moreno A and Rosas-Burgos, Am. J. Phys 76, 1141(2008).

[11] Wilkins D.C, Phys. Rev. D 5, 814(1972).

[12] Kerr R.P, Phys. Rev. Lett. 11, 237(1963).

[13] E.Newman and R.Penrose, J. Math. Phys., 8, 1355(1967).

[14] Chandrasekhar S.(1992), " The Mathematical Theory of Black Holes", Oxford University Press, NewYork.

[15] Krameer D, Stephani H, Herlt E. and MacCallum M.(1980), "Exact Solutions of Einstein Field Equations", Cambridge University Press, Cambridge.

[16] Faridi A. M, Gen. Rel. and Grav, 18, 271(1986).

Samuel J. and Vishweshwara C.V. "Personal Communication".

[17] Ramachandra B. S, Gen. Rel. and Grav, 41, 2757(2009). 
[18] M. Walker and R. Penrose, Commun. Math. Phys., 18, 265(1970).

[19] Carter B, J. Math. Phys., 10, 70(1969).

[20] R. D. Greene, E. L. Schücking and C.V. Vishveshwara, J. Math. Phys. 16, 153 (1975).

[21] M.A. Abramowicz, P.Nurowski and N. Wex, Class Quantum. Grav. 12, 1467 (1995).

[22] Bardeen J. M, Astrophys. J 162, 71(1970).

[23] Misner C.W, Thorne K.S. and Wheeler J.H.(1970), "Gravitation" W. H. Freeman and Company New York. 